\title{
Nonlinear continuous integral-derivative observer
}

\author{
Xinhua Wang and Bijan Shirinzadeh \\ Department of Mechanical and Aerospace Engineering,
}

Monash University, Melbourne, VIC, 3800, Australia Email: wangxinhua04@gmail.com Tel: +610432704528

\begin{abstract}
In this paper, a high-order nonlinear continuous integral-derivative observer is presented based on finite-time stability and singular perturbation technique. The proposed integral-derivative observer can not only obtain the multiple integrals of a signal, but can also estimate the derivatives. Conditions are given ensuring finite-time stability for the presented integral-derivative observer, and the stability and robustness in time domain are analysed. The merits of the presented integral-derivative observer include its synchronous estimation of integrals and derivatives, finite-time stability, ease of parameters selection, sufficient stochastic noises rejection and almost no drift phenomenon. The theoretical results are confirmed by computational analysis and simulations.
\end{abstract}

Key words. Integral-derivative observer, finite-time stability, singular perturbation technique, high-order, drift phenomenon

\section{Introduction}

Time derivatives and integrals are important components in almost all engineering applications. The problems of the time derivatives and integrals are those of estimating the values $D_{i}(a)=\frac{d^{i} a(t)}{d t^{2}}$ and $I(a)=$ $\int_{0}^{t} \cdots \int_{0}^{s} a(\sigma) d \sigma \cdots d \tau$, respectively. Obtaining the positions, velocities and accelerations is crucial for many types of systems for correct and timely performances, such as missile flying control systems [1] and the antilock braking systems [2]. For an airplane, the flying velocity can be obtained from an airspeed indicator. In order to design a feedback controller, the time integral and derivative performances are required to estimate the position and acceleration trajectories of the airplane, respectively. For the guidance of a missile system, the acceleration is obtained from the accelerometer. In order to hit the target, the position trajectory of the missile should be obtained. Therefore, in the circumstances without GPS, we need to compose a double-integral algorithm to estimate the position trajectory from the acceleration measurement on condition that the initial position and velocity are known. Inertial Navigation System (INS) is a selfcontained navigation technique in which measurements provided by accelerometers and gyroscopes are used to track the position and orientation of an object relative to a known starting point, orientation and velocity. In INS, Inertial measurement units (IMUs) typically contain three orthogonal rate-gyroscopes and three orthogonal accelerometers, measuring angular velocity and linear acceleration respectively. To calculate the position of the device the signals from the accelerometers are double integrated. However, for a long-time navigation, the drift phenomenon of INS is mainly brought out by the usual integral methods. They cannot restrain the effect of stochastic noise, especially non-white noise. Such noise leads to the accumulation of additional drift in the integrated signal. Furthermore, the stability analysis for the usual integral methods are seldom given. Therefore, it is important for the design of low-drift integral methods. Importantly, the stability and robustness of integral methods should be analysed.

The algorithms of derivative and integral have been studied by a number of researchers from different perspectives [3]-[20]. In recent years, the observer-like differentiators have been developed [15]-[20], and their stabilities were analysed. Obviously, for the usual observers or differentiators [15]-[20], it is impossible to estimate the integrals of a signal. The usual observers or differentiators can estimate the derivatives of a signal, but not its multiple integrals.

The integral of signal is the infinite sum of differential. The integral of $f(x)$ on $[a, b]$ is defined as $I=$ $\int_{a}^{b} f(x) d x=\lim _{\max \left\{\Delta x_{i} \rightarrow 0\right\}} \sum_{i=1}^{n} f\left(\xi_{i}\right) \Delta x_{i}$, where $a=x_{0}<x_{1}<\cdots<x_{n}=b, \Delta x_{i}=x_{i}-x_{i-1}, \xi_{i} \in\left(x_{i-1}, x_{i}\right)$, $i=1, \cdots, n$. Furthermore, The double integral of $f(x)$ on domain $G$ is defined as $\iint_{G} f(x, y) d x d y=$ $\lim _{\max \left\{\Delta x_{i}^{2}+\Delta y_{i}^{2} \rightarrow 0\right\}} \sum_{i} \sum_{j} f\left(\xi_{i}, \eta_{j}\right) \Delta x_{i} \Delta y_{j}$, where $\Delta x_{i}=x_{i}-x_{i-1}, \Delta y_{j}=y_{j}-y_{j-1}, \xi_{i} \in\left(x_{i-1}, x_{i}\right), \eta_{j} \in$ 
$\left(y_{j-1}, y_{j}\right), i, j=1, \cdots, n$.

At present, there are no general rules for integrating as there are for differentiating. There are some numerical methods for estimating signal integral [12]: i) The trapezoidal rule; ii) Simpson's rule. For the above numerical integrating methods, if stochastic noise (especially non-white noise) exists in signal, and the average value of the noise is not equal to zero, then such noise leads to the accumulation of additional drift in the integrated signal. It is not guaranteed that the integrator is stable. Importantly, it is difficult to analyse the stability and robustness of these methods. Therefore, the choice of integration scheme has a big effect on the performance of a system.

The integral operators of $1 / s$ and $1 / s^{2}$ are irrational and can't be calculated directly. Researchers presented some approximated methods to estimate signal integral [13]-[21]. For the integrators design in frequency domain, an IIR digital integrator was designed by using the Simpson integration rule and fractional delay filter [3]. In [4], a digital IIR integrator based on the recursive Romberg integration rule and the fractional sample delay was investigated. A general theory of the Newton-Cotes digital integrators by applying the z-transform technique to the closed-form Newton-Cotes integration formula was presented [5]. In [7]-[11], several types of digital integrators were designed: non-inverting integrator, the developed infinite impulse response digital integrators, low-frequency differential differentiators. However, in all of the aforementioned studies [3]-[12], the integrators are all linear approximated, only 1-fold integral can be obtained, and the estimations of derivative and integral are not considered synchronously. Importantly, there is no stability analysis. Some integrators were implemented in the hardware, where the parameters are usually affected by the circumstances, for instance, the temperature changes in the circuit. Thus, the estimation precision is affected adversely. Furthermore, they are easily disturbed by stochastic noise, and the drift phenomenon occurs in such systems. In order to restrain the stochastic noise, additional filters must be designed.

In [13] and [14], the concept of fractional-order integrator has been presented, and a rational transfer function is proposed to approximate the irrational fractional-order integrator $G_{I}(s)=1 / \mathrm{s}^{m}$, where $m$ is a positive real number, and $0<m<1$ is required. The condition of $0<m<1$ limits the application of the fractional-order integrator. Usually, the 1-fold and double integrals are required to obtained in many navigation systems, i.e., the operators $1 / s$ and $1 / s^{2}$ are required to be computed. Therefore, this method is not suitable to some navigation systems.

In this paper, a high-order nonlinear integral-derivative observer is presented based on finite-time stability [21]-[28] and singular perturbation technique [29]-[31]. In this derivative-integral observer, a new distribution of perturbation parameters is arranged. Using this integral-derivative observer, the multiple integrals and derivatives of a signal can be obtained synchronously, and the estimation of the time derivatives and integrals is finite-time stable. The integral-chain structure increases filter order and improves the stochastic noise rejection. Importantly, the parameters regulation is only required to be satisfied with Hurwitz condition. The synchronous dynamical performance of time derivatives and integrals is helpful for the design of system controllers (for example, design of proportional-integral-differential (PID) controller) and the guidance methodologies. Furthermore, for the proposed integral-derivative observer, the introduction of a differential feedback term is equivalent that there is a lead compensation (or a dynamic feedback term) in the integral-derivative observer, and the dynamic performance can be improved. Therefore, the drift in the integral outputs can be corrected sufficiently. Importantly, with respect other integrator design, the stability and robustness in time domain of the presented integral-derivative observer are analysed. The closed-loop system is stable.

This paper is organized in the following format. In section 2, preliminaries are introduced. In Section 3 , the main results of the presented integral-derivative observer are presented. In Section 4, the robustness of the proposed integral-derivative observer is analysed. In Section 5, the computational analysis and simulations are described. The conclusions are provided in Section 6.

\section{Preliminaries and background}


The related concepts are presented here.

Definition 1 [22]: Let us consider a time-invariant system in the form of

$$
\dot{x}=f(x), f(0)=0, x \in R^{n},
$$

where $f: D \rightarrow R^{n}$ is continuous on open neighborhood $D \subseteq R^{n}$ of the origin. The origin is said to be a finite-time-stable equilibrium of the above system if there exists an open neighborhood $N \subseteq D$ of the origin and a function $T_{f}: N \backslash\{0\} \rightarrow(0, \infty)$, called the settling-time function, such that the following statements hold: (i) Finite-time-convergence: For every $x \in N \backslash\{0\}, \psi^{x}$ is the flow starting from $x$ and defined on $\left[0, T_{f}(x)\right), \psi^{x}(t) \in N \backslash\{0\}$ for all $t \in\left[0, T_{f}(x)\right)$, and $\lim _{t \rightarrow T_{f}(x)} \psi^{x}(t)=0$.

(ii) Lyapunov stability: For every open neighborhood $U_{\varepsilon}$ of 0 there exists an open subset $U_{\delta}$ of $N$ containing 0 such that, for every $x \in U_{\delta} \backslash\{0\}, \psi^{x}(t) \in U_{\varepsilon}$ for all $t \in\left[0, T_{f}(x)\right)$.

The origin is said to be a globally finite-time-stable equilibrium if it is a finite-time-stable equilibrium with $D=N=R^{n}$. Then the system is said to be finite-time-convergent with respect to the origin.

Assumption 1: For a system depicted by Equation (1), there exists $\rho_{i} \in(0,1], i=1, \cdots, n$, and a nonnegative constant $\bar{a}$ such that

$$
\left|f_{j}\left(\widetilde{z}_{1}, \widetilde{z}_{2}, \cdots, \widetilde{z}_{n}\right)-f_{j}\left(\bar{z}_{1}, \bar{z}_{2}, \cdots, \bar{z}_{n}\right)\right| \leq \bar{a} \sum_{i=1}^{n}\left|\widetilde{z}_{i}-\bar{z}_{i}\right|^{\rho_{i}}
$$

where $\widetilde{z}_{i}, \bar{z}_{i} \in R, i=1, \cdots, n, j=1, \cdots, n$.

Remark 1: There are a number of nonlinear functions capable of satisfying this assumption. For example, one such function is $x^{\rho_{i}}$ since

$$
\left|x^{\rho_{i}}-\bar{x}^{\rho_{i}}\right| \leq 2^{1-\rho_{i}}|x-\bar{x}|^{\rho_{i}}, \rho_{i} \in(0,1]
$$

Moreover, there are smooth functions also satisfying this property. In fact, it is easy to verify that

$$
|\sin x-\sin \bar{x}| \leq 2|x-\bar{x}|^{\rho_{i}}
$$

for any $\rho_{i} \in(0,1]$.

Theorem 4.2 [22]: Suppose there exists a continuous function $V: R^{n}-R$ such that the following conditions holds:

(i) $V$ is positive definite,

(ii) There exist real numbers $c>0$ and $\theta \in(0,1)$ such that

$$
\dot{V}(x)+c(V(x))^{\theta} \leq 0
$$

Then (1) is globally finite-time stable. Moreover, if $N$ is as in Definition 1 and $T_{f}$ is the setting time function then

$$
T_{f}(x) \leq \frac{1}{c(1-\theta)} V(x)^{1-\theta}
$$

Proposition 8.1 [21]: Let $k_{1}, \cdots, k_{n}>0$ be such that $s^{n}+k_{n} s^{n-1}+\cdots+k_{2} s+k_{1}$ is Hurwitz, and consider the system

$$
\begin{aligned}
\dot{x}_{i} & =x_{i+1} ; i=1, \cdots, n-1, \\
\dot{x}_{n} & =-\sum_{i=1}^{n} k_{i}\left|x_{i}\right|^{\alpha_{i}} \operatorname{sign}\left(x_{i}\right)
\end{aligned}
$$


there exists $\xi \in(0,1)$ such that, for every $\alpha \in(1-\xi, 1)$, the origin is globally finite-time-stable equilibrium for Equation (5) where $\alpha_{1}, \cdots, \alpha_{n}$ satisfy

$$
\alpha_{i-1}=\frac{\alpha_{i} \alpha_{i+1}}{2 \alpha_{i+1}-\alpha_{i}}, i=2, \cdots, n
$$

with $\alpha_{n+1}=1$ and $\alpha_{n}=\alpha$.

Theorem 5.2 [22]: Consider the perturbed system of (1) following:

$$
\dot{x}=f(x)+g(t, x(t)), x(0)=x_{0}
$$

Suppose there exists a function $V: D \rightarrow R$ such that $V$ is positive definite and Lipschitz continuous on $D$, and satisfies (3), where $\nu \subseteq D$ is an open neighborhood of the origin, $c>0$ and $\theta \in\left(0, \frac{1}{2}\right)$. Then there exist $\delta_{0}>0, L>0, \Gamma>0$, and an open neighborhood $U$ of origin such that, for every continuous function $g: R_{+} \times D \rightarrow R^{n}$ with

$$
\delta=\sup _{R_{+} \times D}\|g(t, x(t))\| \leq \delta_{0}
$$

every right maximally defined solution $x$ of $(7)$ with $x(0) \in U$ is defined on $R_{+}$and satisfies $x(t) \in U$ for all $t \in R_{+}$and

$$
\|x(t)\| \leq L \delta^{\gamma}, t \geq \Gamma
$$

where $\gamma=(1-\theta) / \theta>1$.

\section{Nonlinear integral-derivative observer}

In the following, finite-time stability [21]-[28] and singular perturbation technique [29]-[31] will be used to design a high-order nonlinear integral-derivative observer, and Theorem 1 is presented as follow.

Theorem 1: For system

$$
\begin{aligned}
\dot{x}_{i}= & x_{i+1} ; i=1, \cdots, n-1 \\
\varepsilon^{n+1} \dot{x}_{n}= & -\sum_{i=1, i \neq p}^{n} k_{i}\left|\varepsilon^{i} x_{i}\right|^{\alpha_{i}} \operatorname{sign}\left(x_{i}\right) \\
& -k_{p}\left|x_{p}-a(t)\right|^{\alpha_{p}} \operatorname{sign}\left(x_{p}-a(t)\right)
\end{aligned}
$$

with $p \in\{2, \cdots, n\}$, if signal $a(t)$ is continuous and $(n-p+1)$ th-order derivable, then there exist $\gamma>1$ and $\Gamma>0$, such that, for $t \geq t_{s}=\varepsilon \Gamma(\Xi(\varepsilon) e(0))$,

$$
\left|x_{i}-a_{i}(t)\right| \leq L \varepsilon^{\alpha_{1} \gamma-i}, i=1, \cdots, n
$$

where $a_{p-i}(t)=\underbrace{\int_{0}^{t} \cdots \int_{0}^{\sigma_{2}}}_{i} a\left(\sigma_{1}\right) \underbrace{d \sigma_{1} \cdots d \sigma_{i}}_{i}, x_{i}(0)=a_{i}(0), i=1, \cdots, p-1 ; x_{p}(0)=a_{p}(0) ; a_{p}(t)=a(t)$; $a_{q}(t)=a^{(q-p)}(t), q=p+1, \cdots, n ; \varepsilon \in(0,1)$ is the perturbation parameter; $L$ is some positive constant; $\sup _{t \in[0, \infty)}\left|a_{i}(t)\right| \leq h_{i}<\infty, i=1, \cdots, n, i \neq p ; \sup _{t \in[0, \infty)}\left|a^{(n-p+1)}(t)\right| \leq L_{a}<\infty ; k_{1}, \cdots, k_{p}, \cdots, k_{n}>0$ are selected such that $s^{n}+k_{n} s^{n-1}+\cdots+\frac{k_{p}}{\varepsilon^{p \alpha_{p}}} s^{p-1}+\cdots+k_{2} s+k_{1}$ is Hurwitz; and $\alpha_{1}, \cdots, \alpha_{n}$ satisfy Equation $(6) ; \gamma=(1-\theta) / \theta, \theta \in\left(0, \alpha_{1} /\left(\alpha_{1}+n+1\right)\right), n \geq 2$; and $\Xi(\varepsilon)=\operatorname{diag}\left\{\varepsilon, \varepsilon^{2}, \cdots, \varepsilon^{n}\right\} . e_{i}=x_{i}-a_{i}(t)$, $i=1, \cdots, n, e=\left[e_{1} \cdots e_{n}\right]^{T}$.

Proof: The system error between system (10) and the derivatives of $a_{1}(t)$ is obtained as follow: 


$$
\begin{aligned}
e_{i}= & e_{i+1} ; i=1, \cdots, n-1 \\
\varepsilon^{n+1} \dot{e}_{n}= & -\sum_{i=1, i \neq p}^{n} k_{i}\left|\varepsilon^{i} e_{i}+\varepsilon^{i} a_{i}(t)\right|^{\alpha_{i}} \\
& \times \operatorname{sign}\left(e_{i}+a_{i}(t)\right) \\
& -\frac{k_{p}}{\varepsilon^{p \alpha_{p}}}\left|\varepsilon^{p} e_{p}\right|^{\alpha_{p}} \operatorname{sign}\left(e_{p}\right) \\
& -\varepsilon^{n+1} a^{(n-p+1)}(t)
\end{aligned}
$$

Thus, Equation (12) can be rewritten as:

$$
\begin{aligned}
\frac{d \varepsilon^{i} e_{i}}{d t / \varepsilon}= & \varepsilon^{i+1} e_{i+1} ; i=1, \cdots, n-1 \\
\frac{d \varepsilon^{n} e_{n}}{d t / \varepsilon}= & -\sum_{i=1, i \neq p}^{n} k_{i}\left|\varepsilon^{i} e_{i}+\varepsilon^{i} a_{i}(t)\right|^{\alpha_{i}} \\
& \times \operatorname{sign}\left(e_{i}+a_{i}(t)\right) \\
& -\frac{k_{p}}{\varepsilon^{p \alpha_{p}}}\left|\varepsilon^{p} e_{p}\right|^{\alpha_{p}} \operatorname{sign}\left(e_{p}\right) \\
& -\varepsilon^{n+1} a^{(n-p+1)}(t)
\end{aligned}
$$

Let a coordinate transformation be described as follows:

$$
\begin{aligned}
\tau & =t / \varepsilon, z_{i}(\tau)=\varepsilon^{i} e_{i}(t), \\
i & =1, \cdots, n, z=\left[z_{1} \cdots z_{n}\right]^{T}, \\
\bar{a}_{i}(\tau) & =\varepsilon^{i} a_{i}(t), i=1, \cdots, n, i \neq p, \\
\bar{a}_{n+1}(\tau) & =\varepsilon^{n+1} a^{(n-p+1)}(t)
\end{aligned}
$$

Therefore, we obtain $z=\Xi(\varepsilon) e$, and Equation (13) can be written as

$$
\begin{aligned}
\frac{d z_{i}}{d \tau}= & z_{i+1} ; i=1, \cdots, n-1 \\
\frac{d z_{n}}{d \tau}= & -\sum_{i=1, i \neq p}^{n} k_{i}\left|z_{i}+\bar{a}_{i}(\tau)\right|^{\alpha_{i}} \operatorname{sign}\left(z_{i}+\bar{a}_{i}(\tau)\right) \\
& -\frac{k_{p}}{\varepsilon^{p \alpha_{p}}}\left|z_{p}\right|^{\alpha_{p}} \operatorname{sign}\left(z_{p}\right)-\bar{a}_{n+1}(\tau)
\end{aligned}
$$

Furthermore, Equation (15) can be rewritten as

$$
\begin{aligned}
\frac{d z_{i}}{d \tau}= & z_{i+1}, i=1, \cdots, n-1 \\
\frac{d z_{n}}{d \tau}= & -\sum_{i=1, i \neq p}^{n} k_{i}\left|z_{i}\right|^{\alpha_{i}} \operatorname{sign}\left(z_{i}\right) \\
& -\frac{k_{p}}{\varepsilon^{p \alpha_{p}}}\left|z_{p}\right|^{\alpha_{p}} \operatorname{sign}\left(z_{p}\right) \\
& -\sum_{i=1, i \neq p}^{n} k_{i}\left\{\left|z_{i}+\bar{a}_{i}(\tau)\right|^{\alpha_{i}} \operatorname{sign}\left(z_{i}+\bar{a}_{i}(\tau)\right)\right.
\end{aligned}
$$




$$
\left.-\left|z_{i}\right|^{\alpha_{i}} \operatorname{sign}\left(z_{i}\right)\right\}-\bar{a}_{n+1}(\tau)
$$

Let

$$
\begin{aligned}
g_{2}(\tau, z(\tau))= & -\sum_{i=1, i \neq p}^{n} k_{i}\left\{\left|z_{i}+\bar{a}_{i}(\tau)\right|^{\alpha_{i}}\right. \\
& \times \operatorname{sign}\left(z_{i}+\bar{a}_{i}(\tau)\right) \\
& \left.-\left|z_{i}\right|^{\alpha_{i}} \operatorname{sign}\left(z_{i}\right)\right\}-\bar{a}_{n+1}(\tau)
\end{aligned}
$$

Therefore, from Assumption 1 and Remark 1, we obtain

$$
\begin{aligned}
\delta & =\sup _{(\tau, z) \in R^{n+1}}\left|g_{2}(\tau, z(\tau))\right| \\
& \leq \sum_{i=1, i \neq p}^{n} 2^{1-\alpha_{i}} k_{i} h_{i}^{\alpha_{i}} \varepsilon^{i \alpha_{i}}+\varepsilon^{n+1} L_{a} \leq \varepsilon^{\rho} \delta_{0}
\end{aligned}
$$

where $\delta_{0}=\sum_{i=1, i \neq p}^{n} 2^{1-\alpha_{i}} k_{i} h_{i}^{\alpha_{i}}+L_{a}$, and

$$
\rho=\min _{i \in\{1, \cdots, n\}, i \neq p}\left\{\min \left\{n+1, i \alpha_{i}\right\}\right\}=\alpha_{1}
$$

In fact, it is checked that the recursive form of Equation (6) may be rewritten in the non-recursive form

$$
\alpha_{i}=\frac{\alpha_{n}}{(n-i+1)-(n-i) \alpha_{n}}, i=1, \cdots, n
$$

We will calculate the minimum value of the following expression:

$$
i \alpha_{i}=\frac{i \alpha_{n}}{(n-i+1)-(n-i) \alpha_{n}}, i=1, \cdots, n
$$

Defining the following function

$$
g_{3}(w)=\frac{w \alpha_{n}}{(n-w+1)-(n-w) \alpha_{n}}, w \in(0, n+1)
$$

and taking derivative of $g_{3}(w)$ with respect to variable $w$, we obtain

$$
\frac{d g_{3}(w)}{d w}=\frac{\alpha_{n}\left[(n+1)-\alpha_{n} n\right]}{\left[(n-w+1)-(n-w) \alpha_{n}\right]^{2}}>0
$$

Because $\alpha_{n} \in(0,1)$, function $g_{3}(w)$ is monotone increasing. Moreover, the sequence $\{1, \cdots, n\}$ is monotone increasing in $(0, n+1)$. Therefore,

$$
\min _{i \in\{1, \cdots, n\}, i \neq p}\left\{i \alpha_{i}\right\}=\alpha_{1}
$$

Furthermore, because $\varepsilon \in(0,1)$ and $\alpha_{i} \in(0,1), i=1, \cdots, n$, we obtain

$$
\max _{i \in\{1, \cdots, n\}, i \neq p}\left\{\varepsilon^{i \alpha_{i}}\right\}=\varepsilon^{\rho}=\varepsilon^{\alpha_{1}}
$$

From Proposition 8.1 in [21], Theorem 5.2 in [22] and Equation (18), there exist positive constants $\mu$ and $\Gamma(z(0))$, such that 


$$
\|z(\tau)\| \leq \mu \delta^{\gamma} \leq \mu\left(\varepsilon^{\alpha_{1}} \delta_{0}\right)^{\gamma}, \forall \tau \in[\Gamma(z(0)), \infty)
$$

Therefore, from coordinate transformation (14), we obtain

$$
\left\|\left[\varepsilon e_{1} \cdots \varepsilon^{n} e_{n}\right]\right\| \leq \mu\left(\varepsilon^{\alpha_{1}} \delta_{0}\right)^{\gamma}, \forall t \in[\varepsilon \Gamma(\Xi(\varepsilon) e(0)), \infty)
$$

Thus, the following inequality holds:

$$
\left|e_{i}\right| \leq \mu\left(\varepsilon^{\alpha_{1}-\frac{i}{\gamma}} \delta_{0}\right)^{\gamma}=L \varepsilon^{\alpha_{1} \gamma-i}, \forall t \in[\varepsilon \Gamma(\Xi(\varepsilon) e(0)), \infty)
$$

where $L=\mu \delta_{0}{ }^{\gamma}$. To make $\alpha_{1} \gamma-i>1, i=1, \cdots, n$, from Theorem 5.2 in [22], we let

$$
\begin{aligned}
\theta & \in\left(0, \min \left\{\alpha_{1} /\left(\alpha_{1}+n+1\right), 1 / 2\right\}\right) \\
& =\left(0, \alpha_{1} /\left(\alpha_{1}+n+1\right)\right)
\end{aligned}
$$

In fact, from Theorem 4.3 in [26], $\theta$ can be chosen to be arbitrarily small. Hence, the requirement that $\theta$ lies on $\theta \in\left(0, \alpha_{1} /\left(\alpha_{1}+n+1\right)\right)$ is not restrictive. Accordingly, we can obtain $\alpha_{1}[(1-\theta) / \theta]-n>1$. Therefore, $\alpha_{1} \gamma-i>1$ for $i=1, \cdots, n$. The choice of $\theta$ leads to $\alpha_{1} \gamma-i>1$ in (27) which implies that for $\varepsilon<1$, the ultimate bound (27) on the estimation error is of higher order than the perturbation. This concludes the proof.

In order to guarantee the stability of integral derivative observer (10), polynomial

$$
s^{n}+k_{n} s^{n-1}+\cdots+\frac{k_{p}}{\varepsilon^{p \alpha_{p}}} s^{p-1}+\cdots+k_{2} s+k_{1}
$$

must be Hurwitz for $\varepsilon \in(0,1)$ and the bounded constants $k_{i}>0, i=1, \cdots, n$. In the following, we will discuss the Routh-Hurwitz Stability Criterion of the polynomial. It will be found that $s^{n}+k_{n} s^{n-1}+\cdots+$ $\frac{k_{p}}{\varepsilon^{p \alpha_{p}}} s^{p-1}+\cdots+k_{2} s+k_{1}$ can't be Hurwitz for arbitrary integers $n$ and $p$ with $\varepsilon \in(0,1)$. Fortunately, for some integers $n$ and $p$, Theorem 1 still holds, and it is satisfied with almost all engineering applications. For instance, the position and velocity can be estimated from the acceleration signal, or position and acceleration can be obtained from the velocity signal.

Lemma 1: The following selections of $n$ and $p$ can make polynomial $s^{n}+k_{n} s^{n-1}+\cdots+\frac{k_{p}}{\varepsilon^{p \alpha_{p}}} s^{p-1}+\cdots+$ $k_{2} s+k_{1}$ Hurwitz, where $\varepsilon \in(0,1)$ and the bounded constants $k_{i}>0, i=1, \cdots, n$ :

a) $n=2$ and $p=2$;

b) $n=3$ and $p \in\{2,3\}$;

c) $n=4$ and $p=3$.

Proof: In the following, for polynomial $s^{n}+k_{n} s^{n-1}+\cdots+\frac{k_{p}}{\varepsilon^{p \alpha_{p}}} s^{p-1}+\cdots+k_{2} s+k_{1}$, we will search for integers $n$ and $p$ to satisfy the Routh-Hurwitz Stability Criterion. It is known that Routh table is the nested structure. Therefore, if there is an integer $N$, when $n=N$, for all $p \in\{2, \cdots, N\}$ and $\varepsilon \in(0,1)$, such that $s^{n}+k_{n} s^{n-1}+\cdots+\frac{k_{p}}{\varepsilon^{p \alpha_{p}}} s^{p-1}+\cdots+k_{2} s+k_{1}$ is not Hurwitz, then when $n \geq N$, this statement still holds.

1) When $n=2$, for polynomial $s^{2}+\bar{k}_{2} s+k_{1}$, the Routh table is

$$
\begin{array}{lll}
s^{2} & 1 & k_{1} \\
s^{1} & \bar{k}_{2} & 0 \\
s^{0} & k_{1} & 0
\end{array}
$$

Obviously, polynomial $s^{2}+\bar{k}_{2} s+k_{1}$ is Hurwitz if $k_{1}>0, \bar{k}_{2}>0$. Therefore, when $n=2, p=2$ and $\bar{k}_{2}=\frac{k_{2}}{\varepsilon^{2 \alpha_{2}}}$, polynomial $s^{2}+\frac{k_{2}}{\varepsilon^{2 \alpha_{2}}} s+k_{1}$ can be Hurwitz for arbitrary $\varepsilon \in(0,1)$. 
2) When $n=3$, for polynomial $s^{3}+\bar{k}_{3} s^{2}+\bar{k}_{2} s+k_{1}$, the Routh table is

$\begin{array}{ccc}s^{3} & 1 & \bar{k}_{2} \\ s^{2} & \bar{k}_{3} & k_{1} \\ s^{1} & \frac{\bar{k}_{3} \bar{k}_{2}-k_{1}}{\bar{k}_{3}} & 0 \\ s^{0} & k_{1} & 0\end{array}$

Polynomial $s^{3}+\bar{k}_{3} s^{2}+\bar{k}_{2} s+k_{1}$ is Hurwitz if $k_{1}>0, \bar{k}_{3}>0$ and $\bar{k}_{2} \bar{k}_{3}>k_{1}$. Therefore, when $n=3, p=3$, $\bar{k}_{2}=k_{2}$ and $\bar{k}_{3}=\frac{k_{3}}{\varepsilon^{3 \alpha_{3}}}$, polynomial $s^{3}+\frac{k_{3}}{\varepsilon^{3 \alpha_{3}}} s^{2}+k_{2} s+k_{1}$ can be Hurwitz for arbitrary $\varepsilon \in(0,1)$; when $n=3$, $p=2, \bar{k}_{2}=\frac{k_{2}}{\varepsilon^{2 \alpha_{2}}}$ and $\bar{k}_{3}=k_{3}$, polynomial $s^{3}+k_{3} s^{2}+\frac{k_{2}}{\varepsilon^{2 \alpha_{2}}} s+k_{1}$ can be Hurwitz for arbitrary $\varepsilon \in(0,1)$.

3) When $n=4$, for polynomial $s^{4}+\bar{k}_{4} s^{3}+\bar{k}_{3} s^{2}+\bar{k}_{2} s+k_{1}$, the Routh table is

$$
\begin{array}{llll}
s^{4} & 1 & \bar{k}_{3} & k_{1} \\
s^{3} & \bar{k}_{4} & \bar{k}_{2} & 0 \\
s^{2} & A_{1} & k_{1} & 0 \\
s^{1} & B_{1} & 0 & 0 \\
s^{0} & k_{1} & 0 & 0
\end{array}
$$

where

$$
A_{1}=\frac{\bar{k}_{4} \bar{k}_{3}-\bar{k}_{2}}{\bar{k}_{4}}, B_{1}=\frac{\frac{\bar{k}_{4} \bar{k}_{3}-\bar{k}_{2}}{\bar{k}_{4}} \bar{k}_{2}-\bar{k}_{4} k_{1}}{\frac{\bar{k}_{4} \bar{k}_{3}-\bar{k}_{2}}{k_{4}}}
$$

Polynomial $s^{4}+\bar{k}_{4} s^{3}+\bar{k}_{3} s^{2}+\bar{k}_{2} s+k_{1}$ is Hurwitz if $k_{1}>0, \bar{k}_{4}>0, \bar{k}_{4} \bar{k}_{3}>\bar{k}_{2}$ and $\bar{k}_{4} \bar{k}_{3} \bar{k}_{2}>\bar{k}_{4}^{2} k_{1}+\bar{k}_{2}^{2}$. Therefore, only when $n=4, p=3, \bar{k}_{2}=k_{2}, \bar{k}_{3}=\frac{k_{3}}{\varepsilon^{3 \alpha_{3}}}$ and $\bar{k}_{4}=k_{4}$, polynomial $s^{4}+k_{4} s^{3}+\frac{k_{3}}{\varepsilon^{3 \alpha_{3}}} s^{2}+k_{2} s+k_{1}$ can be Hurwitz for arbitrary $\varepsilon \in(0,1)$.

4) When $n=5$, for polynomial $s^{5}+\bar{k}_{5} s^{4}+\bar{k}_{4} s^{3}+\bar{k}_{3} s^{2}+\bar{k}_{2} s+k_{1}$, the Routh table is

$$
\begin{array}{llll}
s^{5} & 1 & \bar{k}_{4} & \bar{k}_{2} \\
s^{4} & \bar{k}_{5} & \bar{k}_{3} & k_{1} \\
s^{3} & A_{1} & A_{2} & 0 \\
s^{2} & B_{1} & k_{1} & 0 \\
s^{1} & C_{1} & 0 & 0 \\
s^{0} & k_{1} & 0 & 0
\end{array}
$$

where

$$
\begin{aligned}
A_{1} & =\frac{\bar{k}_{5} \bar{k}_{4}-\bar{k}_{3}}{\bar{k}_{5}}, A_{2}=\frac{\bar{k}_{5} \bar{k}_{2}-k_{1}}{\bar{k}_{5}}, \\
B_{1} & =\frac{\frac{\bar{k}_{5} \bar{k}_{4}-\bar{k}_{3}}{k_{5}} \bar{k}_{3}-\left(\bar{k}_{5} \bar{k}_{2}-k_{1}\right)}{\frac{\bar{k}_{5} \bar{k}_{4}-\bar{k}_{3}}{k_{5}}} \\
C_{1} & =\frac{\frac{\frac{\bar{k}_{5} \bar{k}_{4}-\bar{k}_{3}}{k_{5}} \bar{k}_{3}-\left(\bar{k}_{5} \bar{k}_{2}-k_{1}\right)}{\frac{k_{5} k_{4}-\bar{k}_{3}}{k_{5}}} \frac{\bar{k}_{5} \bar{k}_{2}-k_{1}}{k_{5}}-\frac{\bar{k}_{5} \bar{k}_{4}-\bar{k}_{3}}{k_{5}} k_{1}}{B_{1}}
\end{aligned}
$$

Polynomial $s^{5}+\bar{k}_{5} s^{4}+\bar{k}_{4} s^{3}+\bar{k}_{3} s^{2}+\bar{k}_{2} s+k_{1}$ is Hurwitz if $k_{1}>0, \bar{k}_{5}>0, \bar{k}_{5} \bar{k}_{4}>\bar{k}_{3}, \bar{k}_{5} \bar{k}_{2}>k_{1}, \bar{k}_{4} \bar{k}_{3}+k_{1}>$ $\bar{k}_{5} \bar{k}_{2}+\frac{\bar{k}_{3}^{2}}{k_{5}}$ and $\bar{k}_{3}>\frac{\bar{k}_{5} \bar{k}_{4}-\bar{k}_{3}}{k_{5} k_{2}-k_{1}} k_{1}+\frac{\bar{k}_{5} \bar{k}_{2}-k_{1}}{k_{5} k_{4}-k_{3}} \bar{k}_{5}$. For arbitrary large $\bar{k}_{p}=\frac{k_{p}}{\varepsilon^{p \alpha_{p}}}$ and all $p \in\{2,3,4,5\}$, polynomial $s^{5}+\cdots+\bar{k}_{p} s^{p-1}+\cdots+k_{1}$ can't be Hurwitz. Therefore, for $n=5$, all $p \in\{2,3,4,5\}$ and arbitrary $\varepsilon \in(0,1)$, polynomial $s^{5}+\cdots+\frac{k_{p}}{\varepsilon^{p \alpha_{p}}} s^{p-1}+\cdots+k_{1}$ can't be Hurwitz. 
Accordingly, we find an integer $N=5$, when $n=5$, for all $p \in\{2,3,4,5\}$ and arbitrary large $\frac{k_{p}}{\varepsilon^{p \alpha_{p}}}$, polynomial $s^{5}+\cdots+\frac{k_{p}}{\varepsilon^{p \alpha_{p}}} s^{p-1}+\cdots+k_{1}$ can't be Hurwitz. It is known that Routh table is the nested structure. Therefore, when $n \geq 5$, for all $p \in\{2, \cdots, n\}$ and arbitrary $\varepsilon \in(0,1)$, polynomial $s^{n}+k_{n} s^{n-1}+$ $\cdots+\frac{k_{p}}{\varepsilon^{p \alpha_{p}}} s^{p-1}+\cdots+k_{2} s+k_{1}$ can't be Hurwitz.

\section{Remark 2: Perturbation terms $\varepsilon$ and $\delta$}

From the analysis above, for $\varepsilon \in(0,1)$ and the bounded constants $k_{i}>0, i=1, \cdots, n$, there exist $n$ and $p$, such that polynomial $s^{n}+k_{n} s^{n-1}+\cdots+\frac{k_{p}}{\varepsilon^{p \alpha_{p}}} s^{p-1}+\cdots+k_{2} s+k_{1}$ is Hurwitz. For the following perturbation term in Equation (18)

$$
\delta=\sup _{(\tau, z) \in R^{n+1}}\left|g_{2}(\tau, z(\tau))\right| \leq \varepsilon^{\rho} \delta_{0}
$$

where $\delta_{0}=\sum_{i=1, i \neq p}^{n} 2^{1-\alpha_{i}} k_{i} h_{i}^{\alpha_{i}}+L_{a}$, and

$$
\rho=\min _{i \in\{1, \cdots, n\}, i \neq p}\left\{i \alpha_{i}\right\}=a_{1}
$$

the term $\delta_{0}$ is bounded. Furthermore, $\lim _{\varepsilon \rightarrow 0} \delta=0$.

Remark 3: The following system can also implement the estimation of the time derivatives and integrals:

$$
\begin{aligned}
\dot{x}_{i}= & x_{i+1}, i=1, \cdots, n-1 \\
\varepsilon^{n+1} \dot{x}_{n}= & -\sum_{i=1, i \neq p}^{n} k_{i}\left|\varepsilon^{i} x_{i}\right|^{\alpha_{i}} \operatorname{sign}\left(x_{i}\right) \\
& -k_{p}\left|\varepsilon^{p}\left(x_{p}-a(t)\right)\right|^{\alpha_{p}} \operatorname{sign}\left(x_{p}-a(t)\right)
\end{aligned}
$$

where, $k_{1}, \cdots, k_{n}>0$ are selected such that

$$
s^{n}+k_{n} s^{n-1}+\cdots+k_{2} s+k_{1}
$$

is Hurwitz. However, the error term $\varepsilon^{p}\left(x_{p}-a(t)\right)$ in Equation (36) is very small, and its corresponding error feedback is quite weak. Therefore, the system convergence is slow. In integral-derivative observer (10), the gain $k_{p}$ of the error term $x_{p}-a(t)$ is suitable, thus, the error feedback is more efficient.

From Theorem 1 and Lemma 1, we can obtain the exact forms of integral-derivative observers, and a Corollary is presented as follow.

Corollary 1: There exist the following four types of nonlinear integral-derivative observers:

\section{i. 1-fold-integral observer}

When $n=2$ and $p=2$, Equation (10) can be written as

$$
\begin{aligned}
\dot{x}_{1}= & x_{2} \\
\varepsilon^{3} \dot{x}_{2}= & -k_{1}\left|\varepsilon x_{1}\right|^{\alpha_{1}} \operatorname{sign}\left(x_{1}\right) \\
& -k_{2}\left|x_{2}-a(t)\right|^{\alpha_{2}} \operatorname{sign}\left(x_{2}-a(t)\right)
\end{aligned}
$$

with the conclusion that, for $t \geq t_{s}$,

$$
\left|x_{1}-a_{1}(t)\right| \leq L \varepsilon^{\alpha_{1} \gamma-1},\left|x_{2}-a_{2}(t)\right| \leq L \varepsilon^{\alpha_{1} \gamma-2}
$$


where $a_{1}(t)=\int_{0}^{t} a(\sigma) d \sigma, a_{2}(t)=a(t) ; x_{1}(0)=a_{1}(0), x_{2}(0)=a_{2}(0) ; \varepsilon \in(0,1)$ is the perturbation parameter; $|\dot{a}(t)| \leq L_{a}, L_{a}$ is a positive constant; $k_{1}>0, k_{2}>0 ; \alpha_{1}=\frac{\alpha_{2}}{2-\alpha_{2}}, \alpha_{2} \in(0,1) ; L$ is some positive constant; $\gamma=(1-\theta) / \theta, \theta \in\left(0, \alpha_{1} /\left(\alpha_{1}+3\right)\right)$. It is a 1-fold integral observer, which can obtain the 1-fold integral of signal $a(t)$.

ii. First-order-derivative 1-fold-integral observer

When $n=3$ and $p=2$, Equation (10) can be written as

$$
\begin{aligned}
\dot{x}_{1}= & x_{2} \\
\dot{x}_{2}= & x_{3} \\
\varepsilon^{4} \dot{x}_{3}= & -k_{1}\left|\varepsilon x_{1}\right|^{\alpha_{1}} \operatorname{sign}\left(x_{1}\right) \\
& -k_{2}\left|x_{2}-a(t)\right|^{\alpha_{2}} \operatorname{sign}\left(x_{2}-a(t)\right) \\
& -k_{3}\left|\varepsilon^{3} x_{3}\right|^{\alpha_{3}} \operatorname{sign}\left(x_{3}\right)
\end{aligned}
$$

with the conclusion that, for $t \geq t_{s}$,

$$
\left|x_{i}-a_{i}(t)\right| \leq L \varepsilon^{\alpha_{1} \gamma-i}, i=1,2,3
$$

where $a_{1}(t)=\int_{0}^{t} a(\sigma) d \sigma, a_{2}(t)=a(t), a_{3}(t)=\dot{a}(t) ; x_{1}(0)=a_{1}(0), x_{2}(0)=a_{2}(0) ; \varepsilon \in(0,1)$ is the perturbation parameter; $k_{1}>0, k_{3}>0$ and $k_{2}>\varepsilon^{2 \alpha_{2}} \frac{k_{1}}{k_{3}} ; \alpha_{1}=\frac{\alpha_{2} \alpha_{3}}{2 \alpha_{3}-\alpha_{2}}, \alpha_{2}=\frac{\alpha_{3}}{2-\alpha_{3}}, \alpha_{3} \in(0,1) ; L$ is some positive constant; and $\gamma=(1-\theta) / \theta, \theta \in\left(0, \alpha_{1} /\left(\alpha_{1}+4\right)\right)$. It is an integral-derivative observer, which can obtain the derivative and integral, respectively, of signal $a(t)$.

iii. Double integral observer

When $n=3$ and $p=3$, Equation (10) can be written as

$$
\begin{aligned}
\dot{x}_{1}= & x_{2} \\
\dot{x}_{2}= & x_{3} \\
\varepsilon^{4} \dot{x}_{3}= & -k_{1}\left|\varepsilon x_{1}\right|^{\alpha_{1}} \operatorname{sign}\left(x_{1}\right)-k_{2}\left|\varepsilon^{2} x_{2}\right|^{\alpha_{2}} \operatorname{sign}\left(x_{2}\right) \\
& -k_{3}\left|x_{3}-a(t)\right|^{\alpha_{3}} \operatorname{sign}\left(x_{3}-a(t)\right)
\end{aligned}
$$

with the conclusion that, for $t \geq t_{s}$,

$$
\left|x_{i}-a_{i}(t)\right| \leq L \varepsilon^{\alpha_{1} \gamma-i}, i=1,2,3
$$

where $a_{1}(t)=\int_{0}^{t} \int_{0}^{\sigma_{2}} a\left(\sigma_{1}\right) d \sigma_{1} d \sigma_{2}, a_{2}(t)=\int_{0}^{t} a\left(\sigma_{1}\right) d \sigma_{1}, a_{3}(t)=a(t) ; x_{1}(0)=a_{1}(0), x_{2}(0)=a_{2}(0)$, $x_{3}(0)=a_{3}(0) ; \varepsilon \in(0,1)$ is the perturbation parameter; $k_{1}>0, k_{3}>0$ and $k_{2}>\varepsilon^{3 \alpha_{3}} \frac{k_{1}}{k_{3}} ; \alpha_{1}=\frac{\alpha_{2} \alpha_{3}}{2 \alpha_{3}-\alpha_{2}}$, $\alpha_{2}=\frac{\alpha_{3}}{2-\alpha_{3}}, \alpha_{3} \in(0,1) ; L$ is some positive constant; $\gamma=(1-\theta) / \theta, \theta \in\left(0, \alpha_{1} /\left(\alpha_{1}+4\right)\right)$. It is a double integral observer, which can obtain the 1-fold and double integrals, respectively, of signal $a(t)$.

iv. First-order-derivative double-integral observer

When $n=4$ and $p=3$, Equation (10) can be written as

$$
\begin{aligned}
& \dot{x}_{1}=x_{2} \\
& \dot{x}_{2}=x_{3} \\
& \dot{x}_{3}=x_{4}
\end{aligned}
$$




$$
\begin{aligned}
\varepsilon^{5} \dot{x}_{4}= & -k_{1}\left|\varepsilon x_{1}\right|^{\alpha_{1}} \operatorname{sign}\left(x_{1}\right)-k_{2}\left|\varepsilon^{2} x_{2}\right|^{\alpha_{2}} \operatorname{sign}\left(x_{2}\right) \\
& -k_{3}\left|x_{3}-a(t)\right|^{\alpha_{3}} \operatorname{sign}\left(x_{3}-a(t)\right) \\
& -k_{4}\left|\varepsilon^{4} x_{4}\right|^{\alpha_{4}} \operatorname{sign}\left(x_{4}\right)
\end{aligned}
$$

with the conclusion that, for $t \geq t_{s}$,

$$
\left|x_{i}-a_{i}(t)\right| \leq L \varepsilon^{\alpha_{1} \gamma-i}, i=1,2,3,4
$$

where $a_{1}(t)=\int_{0}^{t} \int_{0}^{\sigma_{2}} a\left(\sigma_{1}\right) d \sigma_{1} d \sigma_{2}, a_{2}(t)=\int_{0}^{t} a\left(\sigma_{1}\right) d \sigma_{1}, a_{3}(t)=a(t), a_{4}(t)=\dot{a}(t) ; x_{1}(0)=a_{1}(0)$, $x_{2}(0)=a_{2}(0), x_{3}(0)=a_{3}(0) ; \varepsilon \in(0,1)$ is the perturbation parameter; $k_{1}>0, k_{4}>0, k_{3}>\varepsilon^{3 \alpha_{3}} \frac{k_{2}}{k_{4}}$ and $k_{2}>\varepsilon^{3 \alpha_{3}} \frac{k_{4}^{2} k_{1}+k_{2}^{2}}{k_{4} k_{3}} ; \alpha_{1}=\frac{\alpha_{2} \alpha_{3}}{2 \alpha_{3}-\alpha_{2}}, \alpha_{2}=\frac{\alpha_{3} \alpha_{4}}{2 \alpha_{4}-\alpha_{3}}, \alpha_{3}=\frac{\alpha_{4}}{2-\alpha_{4}}, \alpha_{4} \in(0,1) ; L$ is some positive constant; $\gamma=(1-\theta) / \theta, \theta \in\left(0, \alpha_{1} /\left(\alpha_{1}+5\right)\right)$. It is an integral-derivative observer, which can obtain the 1-fold, double integrals and first-order derivative, respectively, of signal $a(t)$.

\section{Robustness analysis of nonlinear time integral-derivative observer}

In a realistic problem, signal $a(t)$ in integral-derivative observer system (10) might represent an ideal signal without any disturbance, while stochastic disturbances exist in almost all signals. The following theorem concerns the robustness behavior of the presented integral-observer under bounded perturbations.

Theorem 2: For integral-derivative observer (10), if the disturbance exists in signal $a(t)$, i.e., $a(t)=a_{0}(t)+$ $d(t)$, where $a_{0}(t)$ is the desired signal, $d(t)$ is the bounded stochastic disturbance, and $\sup _{t \in[0, \infty)}|d(t)| \leq$ $L_{d}<\infty$, then there exist $\gamma>1$ and $\Gamma>0$, such that, for $t \geq \varepsilon \Gamma(\Xi(\varepsilon) e(0))$,

$$
\left|x_{i}-a_{0 i}(t)\right| \leq L\left(\delta_{d i}\right)^{\gamma}, i=1, \cdots, n
$$

where

$$
a_{0(p-i)}(t)=\underbrace{\int_{0}^{t} \cdots \int_{0}^{\sigma_{2}}}_{i} a_{0}\left(\sigma_{1}\right) \underbrace{d \sigma_{1} \cdots d \sigma_{i}}_{i}
$$

$x_{i}(0)=a_{0 i}(0), i=1, \cdots, p-1 ; a_{0 p}(t)=a_{0}(t) ; a_{0 q}(t)=a_{0}^{(q-p)}(t), q=p+1, \cdots, n ; L$ is some positive constant; $\delta_{d i}=\varepsilon^{\alpha_{1}-\frac{i}{\gamma}}+\frac{2^{1-\alpha_{p}}}{\delta_{0}} k_{p} L_{d}^{\alpha_{p}} \varepsilon^{-\frac{i}{\gamma}}$, and $\delta_{d i} \in(0,1), i=1, \cdots, n ; \varepsilon \in(0,1)$, and $L_{d}<\left(\frac{1-\varepsilon^{\alpha_{1}}}{2^{1-\alpha_{p}} k_{p}} \delta_{0}\right)^{\frac{1}{\alpha_{p}}}$; $\delta_{0}=\sum_{i=1, i \neq p}^{n} 2^{1-\alpha_{i}} k_{i} h_{i}^{\alpha_{i}}+L_{a} ; \sup _{t \in[0, \infty)}\left|a_{0 i}(t)\right| \leq h_{i}<\infty, i=1, \cdots, n, i \neq p ; \sup _{t \in[0, \infty)}\left|a_{0}^{(n-p+1)}(t)\right| \leq$ $L_{a}<\infty ; \gamma=(1-\theta) / \theta$,

$$
\theta \in\left(0, \min \left\{\frac{1}{\frac{(n+1) \log \varepsilon}{\log \left(\varepsilon^{\alpha_{1}}+\frac{2^{1-\alpha_{p}}}{\delta_{0}} k_{p} L_{d}^{\alpha_{p}}\right)}+1}, \frac{1}{2}\right\}\right), n \geq 2 ;
$$

$\Xi(\varepsilon)=\operatorname{diag}\left\{\varepsilon, \varepsilon^{2}, \cdots, \varepsilon^{n}\right\}$, and $e_{i}=x_{i}-a_{0 i}(t), i=1, \cdots, n, e=\left[e_{1} \cdots e_{n}\right]^{T}$.

Proof: The system error between system (10) and the derivatives of $a_{01}(t)$ is given by:

$$
\begin{aligned}
e_{i}= & e_{i+1} ; i=1, \cdots, n-1 \\
\varepsilon^{n+1} \dot{e}_{n}= & -\sum_{i=1, i \neq p}^{n} k_{i}\left|\varepsilon^{i} e_{i}+\varepsilon^{i} a_{0 i}(t)\right|^{\alpha_{i}} \\
& \times \operatorname{sign}\left(e_{i}+a_{0 i}(t)\right) \\
& -\frac{k_{p}}{\varepsilon^{p \alpha_{p}}}\left|\varepsilon^{p} e_{p}-\varepsilon^{p} d(t)\right|^{\alpha_{p}}
\end{aligned}
$$




$$
\times \operatorname{sign}\left(e_{p}-d(t)\right)-\varepsilon^{n+1} a_{0}^{(n-p+1)}(t)
$$

The Equation (46) can be rewritten as:

$$
\begin{aligned}
\frac{d \varepsilon^{i} e_{i}}{d t / \varepsilon}= & \varepsilon^{i+1} e_{i+1} ; i=1, \cdots, n-1 \\
\frac{d \varepsilon^{n} e_{n}}{d t / \varepsilon}= & -\sum_{i=1, i \neq p}^{n} k_{i}\left|\varepsilon^{i} e_{i}+\varepsilon^{i} a_{0 i}(t)\right|^{\alpha_{i}} \\
& \times \operatorname{sign}\left(e_{i}+a_{0 i}(t)\right) \\
& -\frac{k_{p}}{\varepsilon^{p \alpha_{p}}}\left|\varepsilon^{p} e_{p}-\varepsilon^{p} d(t)\right|^{\alpha_{p}} \\
& \times \operatorname{sign}\left(e_{p}-d(t)\right)-\varepsilon^{n+1} a_{0}^{(n-p+1)}(t)
\end{aligned}
$$

Let

$$
\begin{gathered}
\tau=t / \varepsilon, z_{i}(\tau)=\varepsilon^{i} e_{i}(t), \bar{a}_{i}(\tau)=\varepsilon^{i} a_{0 i}(t), \\
i=1, \cdots, n, z=\left[z_{1} \cdots z_{n}\right]^{T}, \\
\bar{a}_{n+1}(\tau)=\varepsilon^{n+1} a_{0}^{(n-p+1)}(t), \bar{d}(\tau)=\varepsilon^{p} d(t)
\end{gathered}
$$

therefore, we have $z=\Xi(\varepsilon) e$. The Equation (47) can be written as

$$
\begin{aligned}
\frac{d z_{i}}{d \tau}= & z_{i+1} ; i=1, \cdots, n-1 \\
\frac{d z_{n}}{d \tau}= & \sum_{i=1, i \neq p}^{n} k_{i}\left|z_{i}+\bar{a}_{i}(\tau)\right|^{\alpha_{i}} \operatorname{sign}\left(z_{i}+\bar{a}_{i}(\tau)\right) \\
& -\frac{k_{p}}{\varepsilon^{p \alpha_{p}}}\left|z_{p}-\bar{d}(\tau)\right|^{\alpha_{p}} \operatorname{sign}\left(z_{p}-\bar{d}(\tau)\right) \\
& -\bar{a}_{n+1}(\tau)
\end{aligned}
$$

Furthermore, Equation (49) can be rewritten as

$$
\begin{aligned}
\frac{d z_{i}}{d \tau}= & z_{i+1}, i=1, \cdots, n-1 \\
\frac{d z_{n}}{d \tau}= & -\sum_{i=1, i \neq p}^{n} k_{i}\left|z_{i}\right|^{\alpha_{i}} \operatorname{sign}\left(z_{i}\right) \\
& -\frac{k_{p}}{\varepsilon^{p \alpha_{p}}}\left|z_{p}\right|^{\alpha_{p}} \operatorname{sign}\left(z_{p}\right) \\
& -\frac{k_{p}}{\varepsilon^{p \alpha_{p}}}\left\{\left|z_{p}-\bar{d}(\tau)\right|^{\alpha_{p}} \operatorname{sign}\left(z_{p}-\bar{d}(\tau)\right)\right. \\
& \left.-\left|z_{p}\right|^{\alpha_{p}} \operatorname{sign}\left(z_{p}\right)\right\} \\
& -\sum_{i=1, i \neq p}^{n} k_{i}\left\{\left|z_{i}+\bar{a}_{i}(\tau)\right|^{\alpha_{i}} \operatorname{sign}\left(z_{i}+\bar{a}_{i}(\tau)\right)\right. \\
& \left.-\left|z_{i}\right|^{\alpha_{i}} \operatorname{sign}\left(z_{i}\right)\right\}-\bar{a}_{n+1}(\tau)
\end{aligned}
$$

Let

$$
g_{2}(\tau, z(\tau))=-\sum_{i=1, i \neq p}^{n} k_{i}\left\{\left|z_{i}+\bar{a}_{i}(\tau)\right|^{\alpha_{i}}\right.
$$




$$
\begin{aligned}
& \times \operatorname{sign}\left(z_{i}+\bar{a}_{i}(\tau)\right) \\
& \left.-\left|z_{i}\right|^{\alpha_{i}} \operatorname{sign}\left(z_{i}\right)\right\}-\bar{a}_{n+1}(\tau) \\
& -\frac{k_{p}}{\varepsilon^{p \alpha_{p}}}\left\{\left|z_{p}-\bar{d}(\tau)\right|^{\alpha_{p}}\right. \\
& \left.\times \operatorname{sign}\left(z_{p}-\bar{d}(\tau)\right)-\left|z_{p}\right|^{\alpha_{p}} \operatorname{sign}\left(z_{p}\right)\right\}
\end{aligned}
$$

Therefore, from Assumption 1 and Remark 1, we obtain

$$
\begin{aligned}
\delta & =\sup _{(\tau, z) \in R^{n+1}}\left|g_{2}(\tau, z(\tau))\right| \\
& \leq \sum_{i=1, i \neq p}^{n} 2^{1-\alpha_{i}} k_{i} h_{i}^{\alpha_{i}} \varepsilon^{i \alpha_{i}}+\varepsilon^{n+1} L_{a}+2^{1-\alpha_{p}} k_{p} L_{d}^{\alpha_{p}} \\
& \leq \varepsilon^{\rho} \delta_{0}+2^{1-\alpha_{p}} k_{p} L_{d}^{\alpha_{p}}
\end{aligned}
$$

where $\delta_{0}=\sum_{i=1, i \neq p}^{n} 2^{1-\alpha_{i}} k_{i} h_{i}^{\alpha_{i}}+L_{a}$, and

$$
\rho=\min _{i \in\{1, \cdots, n\}, i \neq p}\left\{\min \left\{n+1, i \alpha_{i}\right\}\right\}=\alpha_{1}
$$

From Proposition 8.1 in [21], Theorem 5.2 in [22] and Equation (52), there exist positive constants $\mu$ and $\Gamma(z(0))$, such that

$$
\begin{aligned}
\|z(\tau)\| & \leq \mu \delta^{\gamma} \leq \mu\left(\varepsilon^{\alpha_{1}} \delta_{0}+2^{1-\alpha_{p}} k_{p} L_{d}^{\alpha_{p}}\right)^{\gamma}, \\
\forall \tau & \in[\Gamma(z(0)), \infty)
\end{aligned}
$$

Therefore, from coordinate transformation (48), we obtain

$$
\begin{aligned}
\left\|\left[\varepsilon e_{1} \cdots \varepsilon^{n} e_{n}\right]\right\| & \leq \mu\left(\varepsilon^{\alpha_{1}} \delta_{0}+2^{1-\alpha_{p}} k_{p} L_{d}^{\alpha_{p}}\right)^{\gamma}, \\
\forall t & \in[\varepsilon \Gamma(\Xi(\varepsilon) e(0)), \infty)
\end{aligned}
$$

Thus, the following inequality holds:

$$
\left|e_{i}\right| \leq L\left(\delta_{d i}\right)^{\gamma}, i=1, \cdots, n, \forall t \in[\varepsilon \Gamma(\Xi(\varepsilon) e(0)), \infty)
$$

where $L=\mu \delta_{0}^{\gamma}, \delta_{d i}=\varepsilon^{\alpha_{1}-\frac{i}{\gamma}}+\frac{2^{1-\alpha_{p}}}{\delta_{0}} k_{p} L_{d}^{\alpha_{p}} \varepsilon^{-\frac{i}{\gamma}}, i=1, \cdots, n$. If $\varepsilon \in(0,1)$ and $L_{d}<\left(\frac{1-\varepsilon^{\alpha_{1}}}{2^{1-\alpha_{p}} k_{p}} \delta_{0}\right)^{\frac{1}{\alpha_{p}}}$, then

$$
0<\varepsilon^{\alpha_{1}}+\frac{2^{1-\alpha_{p}}}{\delta_{0}} k_{p} L_{d}^{\alpha_{p}}<1
$$

Furthermore, from Theorem 4.3 in [22], $\theta$ can be chosen to be arbitrarily small. Hence, the requirement that $\theta$ lies on

$$
\theta \in\left(0, \min \left\{\frac{1}{\frac{(n+1) \log \varepsilon}{\log \left(\varepsilon^{\alpha_{1}}+\frac{2^{1-\alpha_{p}}}{\delta_{0}} k_{p} L_{d}^{\alpha_{p}}\right)}+1}, \frac{1}{2}\right\}\right)
$$

is not restrictive. Accordingly, we can obtain $\gamma=(1-\theta) / \theta>\max \left\{\frac{(n+1) \log \varepsilon}{\log \left(\varepsilon^{\alpha_{1}}+\frac{2^{1-\alpha_{p}}}{\delta_{0}} k_{p} L_{d}^{\alpha_{p}}\right)}, 1\right\}$. Therefore, 


$$
\gamma \log \left(\varepsilon^{\alpha_{1}}+\frac{2^{1-\alpha_{p}}}{\delta_{0}} k_{p} L_{d}^{\alpha_{p}}\right)<(n+1) \log \varepsilon
$$

i.e.,

$$
\varepsilon^{\alpha_{1}}+\frac{2^{1-\alpha_{p}}}{\delta_{0}} k_{p} L_{d}^{\alpha_{p}}<\varepsilon^{\frac{n+1}{\gamma}}
$$

Therefore, from $\varepsilon \in(0,1)$ and $\gamma>n+1$, we can obtain $\varepsilon^{\frac{n+1}{\gamma}}<\varepsilon^{\frac{i}{\gamma}}, i=1, \cdots, n$. Then

$$
\delta_{d i}=\varepsilon^{\alpha_{1}-\frac{i}{\gamma}}+\frac{2^{1-\alpha_{p}}}{\delta_{0}} k_{p} L_{d}^{\alpha_{p}} \varepsilon^{-\frac{i}{\gamma}}<1, i=1, \cdots, n
$$

The choice of $\theta$ leads to $\gamma>1$ in (55) which implies that for $\delta_{d i} \in(0,1)$, the ultimate bound (55) on the estimation error is of higher order than the perturbation. Consequently, the presented integral-derivative observer leads to perform rejection of low-level persistent disturbances. This concludes the proof.

\section{Computational analysis and simulations}

In this section, simulation results are presented in order to observe the performances of the proposed integral-derivative observer. We consider the simulations of the following control systems: 1) Integralderivative observer for a input signal; 2) PID control based on integral-derivative observer for a second-order system.

Here, the stochastic non-white noise $\delta(t)$ is selected, and the mean value of the noise is not equal to zero (See the noise in Figure 1(a)). The non-white noise consists of following two signals: Random number with Mean=0, Variance $=0.01$, Initial speed $=0$, and Sample time $=0 ;$ Pulses with Amplitude $=0.5$, Period $=1 \mathrm{~s}$, Pulse width $=1$, and Phase delay $=0$.

\section{1) Integral-derivative observer for a input signal with non-white noise}

For the integral-derivative observer

$$
\begin{aligned}
\dot{x}_{1}= & x_{2} \\
\dot{x}_{2}= & x_{3} \\
\varepsilon^{4} \dot{x}_{3}= & -k_{1}\left|\varepsilon x_{1}\right|^{\alpha_{1}} \operatorname{sign}\left(x_{1}\right) \\
& -k_{2}\left|x_{2}-a(t)\right|^{\alpha_{2}} \operatorname{sign}\left(x_{2}-a(t)\right) \\
& -k_{3}\left|\varepsilon^{3} x_{3}\right|^{\alpha_{3}} \operatorname{sign}\left(x_{3}\right)
\end{aligned}
$$

let the input signal be $a(t)=a_{0}(t)+\delta(t)$, where $a_{0}(t)=\cos t$ is the desired input signal, and $\delta(t)$ is the non-white noise. Therefore, we obtain

$$
\int_{0}^{t} a_{0}(\tau) d \tau=\sin t, \dot{a}_{0}(\tau)=-\sin t
$$

In the integral-derivative observer, $x_{2}$ tracks the reference signal $a_{0}(t) ; x_{1}$ and $x_{3}$ estimate the 1-fold integral and first-order derivative of $a_{0}(t)$, respectively. Observer parameters: $\varepsilon=1 / 2, k_{1}=0.1, k_{2}=2$, $k_{3}=1 ; \alpha_{3}=0.8, \alpha_{2}=\frac{\alpha_{3}}{2-\alpha_{3}}, \alpha_{1}=\frac{\alpha_{2} \alpha_{3}}{2 \alpha_{3}-\alpha_{2}}$. Initial values of observer: $x_{1}(0)=0, x_{2}(0)=1, x_{3}(0)=0$. Signal $a_{0}(t)$ tracking and the estimations of the first-order derivative and 1-fold integral are presented in Figure 1. Figure 1(a) provides signal $a_{0}(t)$ with stochastic noise. Figure 1(b) describes $a_{0}(t)$ tracking. Figures 1(c) and 1(d) present the estimations of the first-order derivative and 1-fold integral, respectively. 
From the above simulations, despite the intensive stochastic noise, the proposed integral-derivative observer showed a very promising tracking ability and robustness.

Furthermore, we compare the presented integral-derivative observer with the integral operator in Matlab Simulink module (See Figures 1(d) and 1(e)). We did the simulation in 3000 seconds, and no drift phenomenon happened for the presented integral-derivative observer (See Figure 1(e)). However, an obvious drift exists in the integral output by the integral operator in Simulink module (See Figure 1(e)). In fact, in MATLAB, some numerical methods are used to estimate signal integral (for example, the trapezoidal rule, Simpson's rule). For the above numerical integrating methods, if white noise exists in signal, the noise can be restrained sufficiently because of integration. However, if stochastic noise (especially non-white noise) exists in signal, and the average value of the noise is not equal to zero, then such noise leads to the accumulation of additional drift in the integrated signal. It is not guaranteed that the system is stable.

2) PID control based on integral-derivative observer for second-order system

The following second-order system is considered:

$$
\begin{aligned}
& \dot{z}_{1}=z_{2} \\
& \dot{z}_{2}=u
\end{aligned}
$$

where, $z_{1}$ and $z_{2}$ are the states, $u$ is the control input. The measurement output is

$$
y=z_{1}+\delta(t)
$$

where $\delta(t)$ is the bounded high-frequency non-white noise. The second-order system is the equivalent or simplified model for many mechanical systems, for instance, inverted pendulum control systems, aircraft attitude control systems, et al.

We are interested in designing a PID controller $u$ to force the system to asymptotically track a given reference signal without the information of $z_{2}$ and $\int_{0}^{t} z_{1}(\tau) d \tau$.

Let the reference trajectory be $\left(z_{d}, \dot{z}_{d}\right)$. The goal of control is that

$$
z_{1} \rightarrow z_{d}, z_{2} \rightarrow \dot{z}_{d}
$$

as $t \rightarrow \infty$. For the reference trajectory $\left(z_{d}, \dot{z}_{d}\right)$, let $e_{1}=z_{1}-z_{d}$ and $e_{2}=z_{2}-\dot{z}_{d}$. The system error is

$$
\begin{aligned}
& \dot{e}_{1}=e_{2} \\
& \dot{e}_{2}=u-\ddot{z}_{d}(t)
\end{aligned}
$$

If $z_{1}, z_{2}$ and $\int_{0}^{t} z_{1}(\tau) d \tau$ are all known, the PID controller can be designed as:

$$
u=K_{P} e_{1}+K_{I} \int_{0}^{t} e_{1}(\tau) d \tau+K_{D} \dot{e}_{1}+\ddot{z}_{d}(t)
$$

Therefore, the closed-loop error system is

$$
\begin{aligned}
& \dot{e}_{1}=e_{2} \\
& \dot{e}_{2}=K_{P} e_{1}+K_{I} \int_{0}^{t} e_{1}(\tau) d \tau+K_{D} \dot{e}_{1}(t)
\end{aligned}
$$

Let 


$$
w_{1}=\int_{0}^{t} e_{1}(\tau) d \tau, w_{2}=e_{1}(t), w_{3}=e_{2}(t)
$$

Therefore, it follows that

$$
\begin{aligned}
& \dot{w}_{1}=w_{2} \\
& \dot{w}_{2}=w_{3} \\
& \dot{w}_{3}=K_{I} w_{1}+K_{P} w_{2}+K_{D} w_{3}
\end{aligned}
$$

The parameters $K_{I}, K_{P}$ and $K_{D}$ are selected such that $s^{3}+K_{D} s^{2}++K_{P} s+K_{I}$ is Hurwitz, then the closed-loop system is stable.

However, $z_{2}$ and $\int_{0}^{t} z_{1}(\tau) d \tau$ are unknown, and the non-white noise $\delta(t)$ exists in the measurement output $y=z_{1}+\delta(t)$. Here, the presented integral-derivative observer is used to estimate these unknown variables from the measurement output $y$, and the noise $\delta(t)$ is reduced sufficiently. The integral-derivative observer is designed as

$$
\begin{aligned}
\dot{x}_{1}= & x_{2} \\
\dot{x}_{2}= & x_{3} \\
\varepsilon^{4} \dot{x}_{3}= & -k_{1}\left|\varepsilon x_{1}\right|^{\alpha_{1}} \operatorname{sign}\left(x_{1}\right) \\
& -k_{2}\left|x_{2}-y\right|^{\alpha_{2}} \operatorname{sign}\left(x_{2}-y\right) \\
& -k_{3}\left|\varepsilon^{3} x_{3}\right|^{\alpha_{3}} \operatorname{sign}\left(x_{3}\right)
\end{aligned}
$$

where $x_{2}$ tracks the state $z_{1} ; x_{1}$ and $x_{3}$ estimate the integral and derivative of state $z_{1}$, respectively.

The PID controller is designed as

$$
u=K_{P} \widehat{e}_{2}+K_{I} \widehat{e}_{1}+K_{D} \widehat{e}_{3}+\ddot{z}_{d}(t)
$$

where $\widehat{e}_{2}=x_{2}-z_{d}(\tau), \widehat{e}_{1}=x_{1}-\int_{0}^{t} z_{d}(\tau) d \tau, \widehat{e}_{3}=x_{3}-\dot{z}_{d}(\tau)$.

Let the reference trajectory $\left(z_{d}, \dot{z}_{d}\right)=(\cos t,-\sin t)$. Therefore, $\int_{0}^{t} z_{d}(\tau) d \tau=\sin t$ and $\ddot{z}_{d}=-\cos t$.

Observer parameters: $\varepsilon=1 / 3, k_{1}=0.1, k_{2}=2, k_{3}=1, \alpha_{3}=0.9, \alpha_{2}=\frac{\alpha_{3}}{2-\alpha_{3}}, \alpha_{1}=\frac{\alpha_{2} \alpha_{3}}{2 \alpha_{3}-\alpha_{2}}$; the initial value of the second system is $\left(z_{1}(0)=0.5, z_{2}(0)=-0.5\right)$; the initial value of the observer is $\left(x_{1}(0)=0\right.$, $\left.x_{2}(0)=0.5, x_{3}(0)=-0.5\right)$; controller parameters: $K_{P}=-2, K_{I}=-1, K_{D}=-1$.

Figure 2 shows the trajectory tracking and the estimations of derivative and integral for the second-order system. Figure 2(a) describes the measurement output $y$ and noise; Figure 2(b) describes the estimation and tracking of $z_{1}$; Figure $2(\mathrm{c})$ describes the estimation of $\int_{0}^{t} z_{1}(\tau) d \tau$; Figure $2(\mathrm{~d})$ describes the estimation and tracking of $z_{2}$; Figure $2(\mathrm{e})$ presents the controller $u$. In the simulation above, though stochastic noises exist in the measurement output, the estimations by the presented integral-derivative observer and the control results by the designed PID controller have satisfying qualities. Even in long-time simulation, no drift phenomenon happen, and the estimations are accurate. However, from Figure 2(c), an obvious drift exists in the integral output by the integral operator in Simulink module. The integral algorithm can't restrain the effect of stochastic noise (especially non-white noise). Such noise leads to the accumulation of additional drift in the integrated signal.

\section{Conclusions}

In this paper, a nonlinear integral-derivative observer based on finite-time stability is presented. The proposed integral-derivative observer can estimate the integrals and derivatives of a signal synchronous. The 
parameters selection is only required to be satisfied with Hurwitz condition. Furthermore, the integralderivative observer exhibits excellent robustness and dynamical performance, and almost without drift phenomenon.

\section{Acknowledgements}

This research is supported in part by Australian Research Council (ARC) Discovery (Grant nos. DP 0986814, DP 110104970), ARC linkage infrastructure, Equipment and Facilities (Grant nos. LE 0347024, LE 0668508).

\section{References}

[1] Takehira, T., Vinh, N.X., Kabamba, P.T.: Analytical solution of missile terminal guidance. AIAA Guidance, Navigation, and Control Conference, New Orleans, LA, United States, 11-13, 172-178 (1997)

[2] Drakunov, V.S., Ozguner, Dix, P., Ashrafi, B.: ABS control using optimum search via sliding modes. IEEE Transactions on Control Systems Technology, 3(1). 79-85 (1995)

[3] Tseng, C.C.: Digital integrator design using Simpson rule and fractional delay filter. IEE Proceedings - Vision, Image and Signal Processing, 153(1), 79-86 (2006)

[4] Tseng, C.C., Lee, S.L.: Digital IIR integrator design using recursive Romberg integration rule and fractional sample delay. Signal Processing, 88(9), 2222-2233 (2008)

[5] Ngo, N.Q.: A new approach for the design of wideband digital integrator and differentiator. IEEE Transactions on Circuits and Systems II: Express Briefs, 53(9), 936-940 (2006)

[6] Hodges, T., Nelson, P.A., Elliott, S.J.: The design of a precision digital integrator for use in an active vibration control system. Mechanical Systems and Signal Processing, 4(4), 345-353 (1990)

[7] Al-Alaoui, M.A.: A novel approach to designing a noninverting integrator with built-in low frequency stability, high frequency compensation, and high Q. IEEE Transactions on Instrumentation and Measurement, 38(6), 1116-1121 (1989)

[8] Al-Alaoui, M.A.: Novel digital integrator and differentiator. Electronics Letters, 29(4), 376-378 (1993)

[9] Al-Alaoui, M.A.: A Class of Second Order Integrators and Lowpass Differentiators. IEEE Transactions on Circuits and Systems-I: Fundamental Theory and Applications, 42(4), 220-223 (1995)

[10] Al-Alaoui, M.A.: Low-frequency differentiators and integrators for biomedical and seismic signals, IEEE Transactions on Circuits and Systems-I: Fundamental Theory and Applications, 48(8), 1006-1011 (2001)

[11] Al-Alaoui, M.A.: Class of digital integrators and differentiators. IET Signal Process. 5(2), 251-260 (2011)

[12] Hahn, B.H., Valentine, D.T.: Essential MATLAB for Engineers and Scientists, 4nd ed Elsevier Ltd. (2010)

[13] Charef, A., Sun, H.H., Tsao, Y.Y., Onaral, B.: Fractal system as represented by singularity function, IEEE Transactions on Automatic Control, 37(9), 1465-1470 (1992)

[14] Chiaref, A.: Analogue realisation of fractional-order integrator, differentiator and fractional $P I^{\lambda} D^{\mu}$ controller, IEE Proc. - Control Theory Appl., 153(6), 714-720 (2006) 
[15] Pei, S.C., Shyu, J.J.: Design of FIR Hilbert Transformers and differentiators by eigenfilter. IEEE Trans. Acoust. Speech Signal Process., ASSP(37), 505-511 (1989)

[16] Atassi, A.N., Khalil, H.K.: Separation results for the stabilization of nonlinear systems using different high-gain observer designs. Systems and Control Letters, 39, 183-191 (2000)

[17] Levant, A.: High-order sliding modes, differentiation and output-feedback control. International Journal of Control, 76(9/10), 924-941 (2003)

[18] Wang, X., Chen, Z., Yang, G.: Finite-time-convergent differentiator based on singular perturbation technique. IEEE Transactions on Automatic Control, 52(9), 1731-1737 (2007)

[19] Wang, X., Shirinzadeh, B.: Rapid-convergent nonlinear differentiator. Mechanical Systems and Signal Processing, 28, 414-431 (2012)

[20] Efimov, D. V., Fridman, L.: A hybrid robust non-homogeneous finite-time differentiator. IEEE Transactions on Automatic Control, 56(5), 1213-1219 (2011)

[21] Bhat, S.P., Bernstein, D.S.: Geometric homogeneity with applications to finite-time stability. Mathematics of Control, Signals, and Systems, 17, 101-127 (2005)

[22] Bhat, S.P., Bemstein, D.S.: Finite-time stability of continuous autonomous systems. Siam J. Control Optim., 38(3), 751-766 (2000)

[23] Haimo, V.T.: Finite time controllers. Siam J. Control Optim., 24(4), 760-771 (1986)

[24] Li, S., Du, H., Lin, X.: Finite-time consensus algorithm for multi-agent systems with double-integrator dynamics. Automatica, 47, 1706-1712 (2011)

[25] Sun, H., Li, S., Sun, C.: Finite time integral sliding mode control of hypersonic vehicles, Nonlinear Dynamics, 73(1-2), 229-244 (2013)

[26] Hu, Q., Li, B., Zhang, A.: Robust finite-time control allocation in spacecraft attitude stabilization under actuator misalignment, Nonlinear Dynamics, 73,(1-2), 53-71 (2013)

[27] Aghababa, M. P., Aghababa, H.P.: Chaos suppression of rotational machine systems via finite-time control method, Nonlinear Dynamics, 69(4), 1881-1888 (2012)

[28] Guo, Z., Huang, L.: Global exponential convergence and global convergence in finite time of nonautonomous discontinuous neural networks, Nonlinear Dynamics, 58(1-2), 349-359 (2009)

[29] Isidori, A., Sastry, S.S., Kokotovic, P.V., Byrnes, C. I.: Singular perturbed zero dynamics of nonlinear systems, IEEE Trans. Automat. Contr., 37, 1625-1631 (1992)

[30] Lee, J.I., Ha, I.J.: A novel approach to control of nonminimum-phase nonlinear systems, IEEE Trans. Automat. Contr., 47, 1480-1486 (2002).

[31] Kahlil, H.K.: Nonlinear systems, 3nd ed. Englewood Cliffs, New Jerse: Prentice-Hall (2002) 

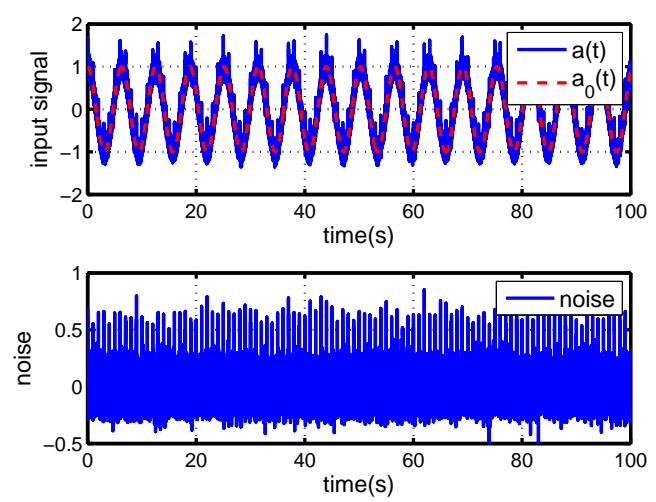

1(a) Input signal
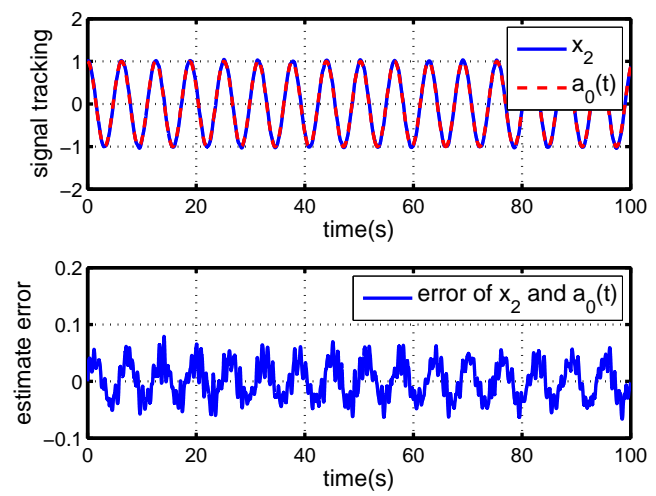

1(b) $a_{0}(t)$ tracking
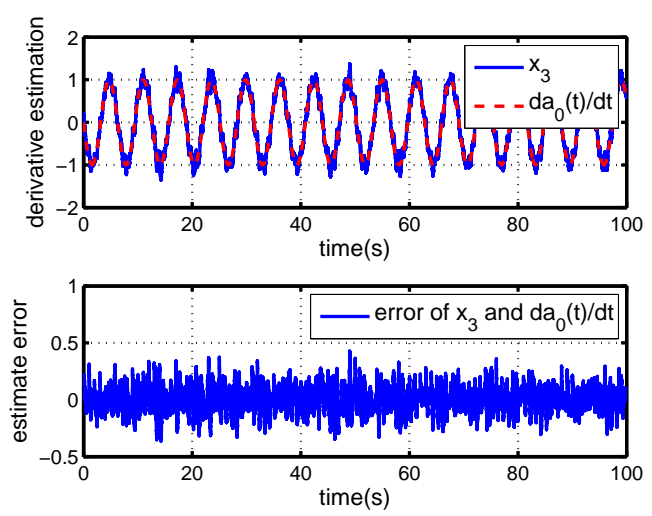

1(c) Derivative estimate
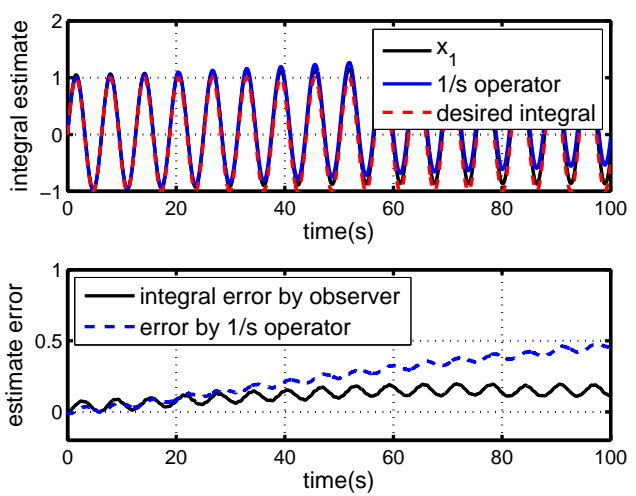

1(d) Integral estimate in 100s 

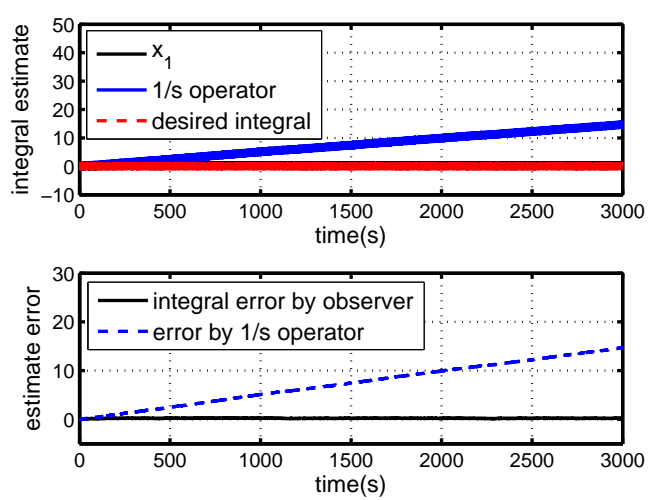

1(e) Integral estimate in $3000 \mathrm{~s}$

Figure 1 Integral-derivative observer
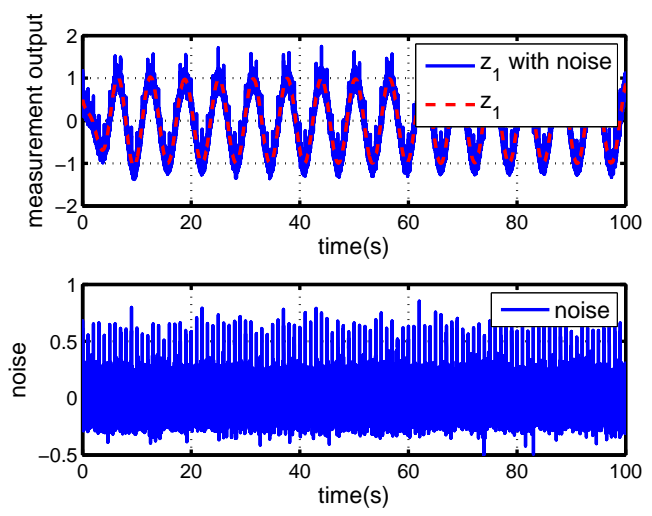

2(a) Measurement output
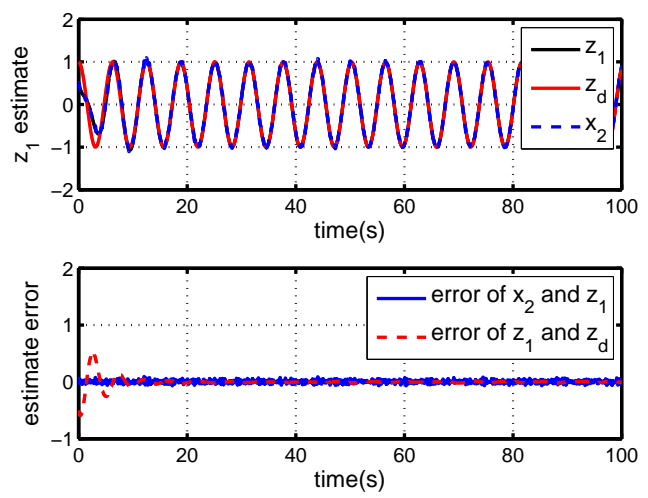

2(b) $z_{1}$ estimate
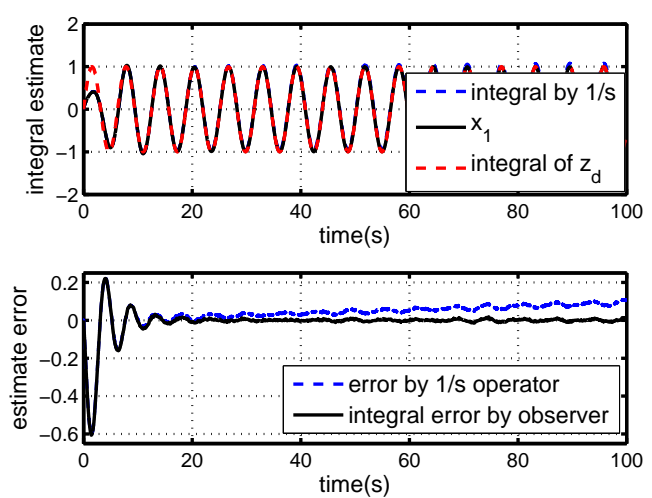

2(c) Estimate of integral of $z_{1}$ 

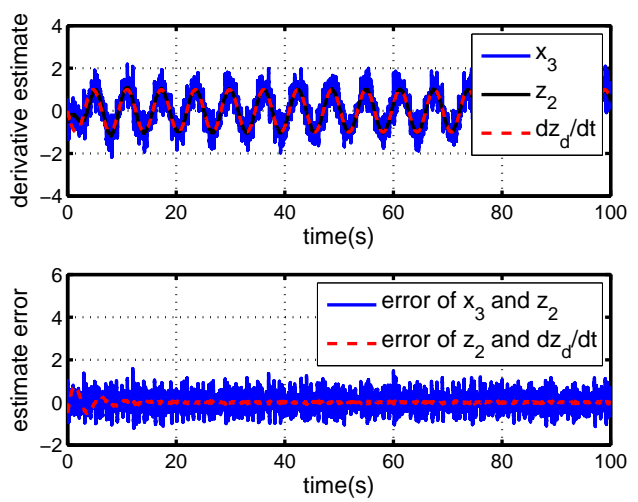

$2(d) z_{2}$ estimate

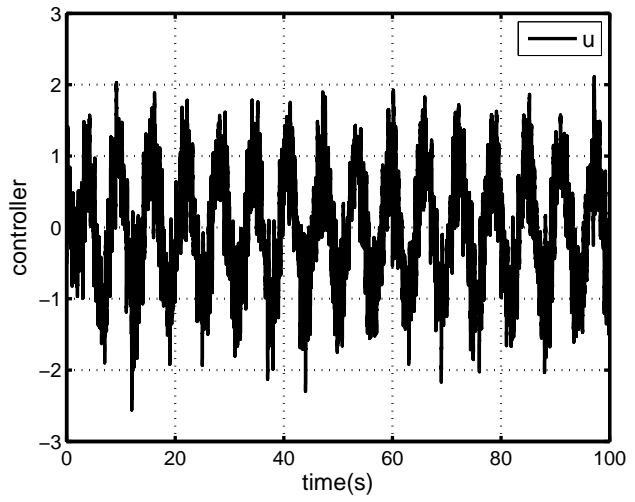

2(e) Controller $u$

Figure 2 PID control based on integral-derivative observer 